\title{
CORRESPONDENCE/
}

\section{Vaccine Crosstalk}

To the editor:

The July 1992 feature "Biotech Vaccines' Problematic Promise" (Stephen M. Edgington, p. 763-766) contained two errors of omission and one error of inclusion of outdated information regarding the status of therapeutic vaccine development at IDEC Pharmaceuticals Corporation.

Our HIV therapeutic vaccine is further along in development than your story indicated. In February 1992, IDEC Pharmaceuticals 3C9, an anti-idiotypic monoclonal antibody, became the first product of its kind in the U.S. to enter phase I clinical trials against HIV infection. IDEC Pharmaceuticals designed $3 \mathrm{C} 9$ as a surrogate antigen to trigger the patient's immune system to produce antibodies to the CD4 binding site on HIV, and thereby neutralize the ability of multiple strains of $\mathrm{HIV}$ to bind and enter CD4+ cells. By targeting a structurally conserved region of the virus, we hope to overcome the clinical problems associated with rapid mutation of HIV.

Our two candidates for treatment of malignant melanoma were omitted from the chart entirely. Over 100 patients at seven research centers in the U.S. have been treated with our I-Mel-1 or I-Mel-2 antiidiotypic vaccines in phase II/ III clinical trials. At a recent NATOAdvanced Studies conference in Greece, we reported that in the serum of the subset of patients we have analyzed so far, both I-Mel-1 and I-Mel-2 monoclonal antibodies stimulate the production of anti-melanoma antibodies in a significant majority of the patients tested.

IDEC Pharmaceuticals' approach to "biotech vaccines" differs somewhat from most other products listed in your table. Rather than using recombinant antigens, we are developing immunologically active monoclonal antibodies for therapeutic applications. Potentially, they offer greater specificity of action, longer therapeutic effect and lower toxicity than is typical of existing therapies.

Richard W. Krawiec Director, Investor Relations \& Corporate Communications IDEC Pharmaceuticals La Jolla, CA 92037

P.S.-I spoke with Tom Cooper who prepared the data at Bioindex. I will provide him with updates of our activities, so that his database remains current.

\section{To the editor:}

Stephen Edgington's article, "Biotech Vaccines' Problematic Promise" [Bio/Technology 10:766 (1992)] was generally informative, but his discussion of FDA's regulation of vaccines was inaccurate and confusing.

Edgington is muddled on what applications must be submitted to FDA for various products. He says that for vaccines "instead of filing a single investigational new drug application (IND), as with therapeutics, [FDA's Center for Biologics
Evaluation and Research] requires two application filings: a product license application (PLA), similar to an IND, and an establishment license application (ELA), a manufacturing license for the vaccine." Actually, whether a product is a "therapeutic" does not dictate where it is regulated or its regulatory requirements. More significant from a regulatory standpoint is whether a product is a "drug" or a "biological" product [see, e.g., H.I. Miller, Bio/Technology 6:1385 (1988)].

Sponsors of investigations on new drugs or biologicals (or new uses of approved drugs or biologicals) must file an IND application, which must include information on the product's composition, manufacturing and controls data, animals test results, training and experience of the investigators, and a plan for the clinical investigation. Data generated in successful clinical trials of a drugbecome the substance of a New Drug Application (NDA) to FDA for marketing approval. Among other required information, the NDA must contain a description and analysis of data and other information that bears on the drug's safety and effectiveness.

A biological product (biologic) is "any virus, therapeutic serum, toxin, antitoxin, vaccine, blood, blood component or derivative, allergenic product, or analogous product . . . applicable to the prevention, treatment, or cure of diseases or injuries of man...." During the IND phase, FDA applies the same regulations to biologics as to drugs. But for marketing approval of a biologic, both the product and its manufacturing facility require licenses. The manufacturer submits a Product License Application (PLA) for the product and an Establishment $\mathrm{Li}$ cense Application (ELA) for the facility. Each must meet

standards designed to ensure the product's safety, purity, potency, and efficacy. FDA monitors each lot of licensed product released.

Edgington asserts that "unlike therapeutic drug-makers, vaccine-makers fall under the jurisdiction of the Center for Biologics Evaluation and Research" (CBER). In fact, well over $90 \%$ of the more than 1100 INDs submitted to FDA for new biotechnology-derived therapeutic products reside in CBER because the products are biologics. These important products include the interferons, $\mathrm{PAA}$, colony stimulating factors, monoclonal antibodies for therapy, clotting factors, and so forth.

Edgington notes that, "while purity and potency are quantifiable, safety is the hinge on which the door of acceptance or rejection swings." Safety is also quantifiable, and the hundreds of thousands of pages of safety data submitted to FDA and its ordering by statisticians is testimony to that. Also, as enumerated above, the requirements for marketing approval include assurance of purity, potency, and efficacy, as well as safety; and approval "hinges" on all of these criteria. Determinations by FDA reviewers of these risk/benefit balances are seldom easy, but they are the FDA's everyday responsibility.

The nuances of FDA's regulatory procedures and requirements may sometimes be elusive, but our individual centers and the Office of Biotechnology are always available to re- 